\title{
New Endovascular Strategy to Overcome Anatomical Constraints When Dealing With Aortoiliac Aneurysms
}

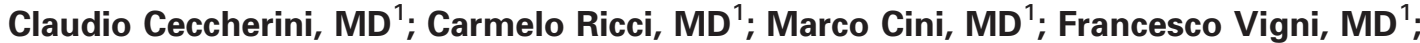 \\ Sara Leonini, MD'; Giulio Tommasino, MD²; Luigi Muzzi, MD²; Enrico Tucci, MD²; \\ Antonio Benvenuti, $\mathrm{MD}^{2}$; and Eugenio Neri, $\mathrm{MD}^{2}$ \\ ${ }^{1}$ Interventional Radiology and ${ }^{2}$ Cardiovascular Surgery, Policlinico Santa Maria alle Scotte, \\ Azienda Ospedaliera Universitaria Senese, Siena, Italy.
}

Involvement of the iliac arteries with an abdominal aortic aneurysm (AAA) is seen in $20 \%$ to $30 \%$ of AAA patients. ${ }^{1}$ Treatment options have been dramatically changed over the last 10 years. At first, the only endovascular option was embolization of the internal iliac artery (IIA) using coils or plugs to extend the iliac limbs of the aortic stent-graft past the IIA. In a significant number of patients, however, IIA embolization may cause chronic symptoms, such as buttock claudication and sexual dysfunction, whether transient or permanent. The most successful endovascular options to preserve IIA flow have been branched stent-grafts and the bell-bottom technique. Implantation of branched stentgrafts has been shown to be feasible and safe, with good long-term outcome, ${ }^{2}$ even if the device is significantly more expensive. The bell-bottom technique was developed to approach IIAs with diameters between 18 and $24 \mathrm{~mm}$, which precludes its use in aneurysms with larger distal landing zones. ${ }^{3}$

In 2011, Lobato et al. ${ }^{1}$ described the sandwich technique for aortoiliac aneurysms, which included 5 steps: (1) bifurcated stentgraft main body insertion through an ipsilateral femoral approach and positioned such that the distal end of the iliac limb is $1 \mathrm{~cm}$ above the IIA origin; (2) catheterization of the ipsilateral IIA through a left brachial access; (3) placement of a covered self-expanding stent $2 \mathrm{~cm}$ inside the IIA with a 6 -cm overlap into the iliac limb, followed by positioning of an iliac limb extension $1 \mathrm{~cm}$ below the covered stent's proximal end; (4) modeling of the iliac limb stent-grafts using a latex balloon and dilation of the covered stent with an angioplasty balloon; and (5) deployment of the contralateral iliac limb. As performed by the authors, the sandwich technique was favorably accepted by other investigators, ${ }^{4}$ and midterm results seem promising. ${ }^{5}$

In our institution, we have used this technique in 12 patients to date ( 7 described in a previous article ${ }^{6}$ ). Cannulating the IIA from the brachial artery and advancing an endograft from the upper extremity proved uncomplicated. Sealing the commissural angles was successful, as oversizing the limb grafts in relation to the diameter of the main graft produces a tight apposition of the components. ${ }^{7}$ The technique satisfactorily recanalized the IIA and was free from intraprocedural complications. Now with a mean follow-up of 20 months (range 6-47), we have seen no endoleak in any patient. The iliac aneurysm sac diameter has shrunk (range 1-15 mm) in two thirds of the common iliac artery (CIA) aneurysms and is unchanged in the other

Invited commentaries published in the Journal of Endovascular Therapy reflect the opinions of the author(s) and do not necessarily represent the views of the Journal or the INTERNATIONAL SOCIETY OF ENDOVASCULAR SPECIALISTS.

The author declares no association with any individual, company, or organization having a vested interest in the subject matter/products mentioned in this article.

Corresponding author: Claudio Ceccherini, MD, Radiologia Interventistica, Policlinico Santa Maria alle Scotte, Azienda Ospedaliera Universitaria Senese, viale Bracci 16, 53100 Siena, Italy. E-mail: claudiocec@hotmail.it 
third; no sac expansion has been seen, and there has been no buttock claudication at last follow-up.

Despite these promising experiences, we noted some disadvantages of the sandwich technique: (1) it requires a brachial artery access (in addition to the femoral artery accesses needed in branched iliac stentgrafts) and a larger introducer (usually 9-F); (2) the Viabahn stent-grafts are not very radiopaque, so positioning them requires extra experience; (3) it is an expensive procedure; and (4) the maximum available diameter of the Viabahn stent-graft is $13 \mathrm{~mm} .^{6}$

The crossover chimney technique described by Wu et al. ${ }^{8}$ in this issue of the JEVT is intriguing and innovative. It consists of delivering the chimney graft across the aortic bifurcation outside the bifurcated stent-graft to provide retrograde flow to the IIA. This technique, which appears relatively easy in distal aortas of normal caliber, could be used regardless of the $\mathrm{CIA}$ dimensions or the presence of endoluminal thrombus (unlike the bell-bottom and branch techniques). It can be performed in association with most types of abdominal endoprostheses (not possible with the branch technique), and there is no additional cost for a branched device or need for percutaneous brachial artery access, as with the sandwich technique. This last point seems the main advantage of this procedure, especially when considering brachial access site-related complications, such as thrombosis or pseudoaneurysm, which typically require surgical correction. On the other hand, we suggest using the sandwich technique in patients with bilateral IIAs because it seems a faster procedure.

In conclusion, more experience with this new technique is certainly warranted, and longer follow-up is necessary before making true comparisons with other techniques.

\section{REFERENCES}

1. Lobato AC. Sandwich technique for aortoiliac aneurysms extending to the internal iliac artery or isolated common/internal iliac artery aneurysms: a new endovascular approach to preserve pelvic circulation. J Endovasc Ther. 2011; 18:106-111.

2. Parlani G, Verzini F, De Rango $P$, et al. Long-term results of iliac aneurysm repair with iliac branched endograft: a 5-year experience on 100 consecutive cases. Eur J Vasc Endovasc Surg. 2012;43:287-292.

3. Torsello G, Schönefeld E, Osada N, et al. Endovascular treatment of common iliac aneurysms using the bell-bottom technique. J Endovasc Ther. 2010;17:504-509.

4. Parodi JC, Parodi FE. Commentary: The sandwich technique to preserve the hypogastric artery during EVAR. J Endovasc Ther. 2011;18: 112-113.

5. Lobato AC, Camacho-Lobato L. The sandwich technique to treat complex aortoiliac or isolated iliac aneurysms: results of midterm follow-up. $J$ Vasc Surg. 2013;57(2 Suppl):26S-34S.

6. Ricci C, Ceccherini $C$, Cini M, et al. Single-center experience and 1-year follow-up results of "sandwich technique" in the management of common iliac artery aneurysms during EVAR. Cardiovasc Intervent Radiol. 2012;35:1195-1200.

7. Falkensammer J, Hakaim AG, Oldenburg WA, et al. Natural history of the iliac arteries after endovascular abdominal aortic aneurysm repair and suitability of ectatic iliac arteries as a distal sealing zone. J Endovasc Ther. 2007;14:619-624.

8. $\mathrm{Wu} I \mathrm{H}$, Chan CY, Chen YS, et al. Crossover chimney technique to preserve the internal iliac artery in abdominal aortic aneurysm with common iliac artery aneurysms. J Endovasc Ther. 2013;20:000-000. 